\title{
New Group Chain Sampling Plan (NGChSP-1) for Generalized Exponential Distribution
}

\author{
Nazrina Aziz ${ }^{1,2,}$, Tan Jia Xin ${ }^{1}$, Zakiyah Zain ${ }^{1,2}$, Mohd Azri Pawan Teh ${ }^{1}$ \\ ${ }^{1}$ Department of Mathematics \& Statistics, School of Quantitative Sciences, Universiti Utara Malaysia, 06010 UUM Sintok, Kedah, \\ Malaysia \\ ${ }^{2}$ Institute of Strategic Industrial Decision Modelling (ISIDM), Universiti Utara Malaysia, 06010 UUM Sintok, Kedah, Malaysia
}

Received August 27, 2021; Revised December 13, 2021; Accepted January 4, 2022

\section{Cite This Paper in the following Citation Styles}

(a): [1] Nazrina Aziz, Tan Jia Xin, Zakiyah Zain, Mohd Azri Pawan The, "New Group Chain Sampling Plan (NGChSP-1) for Generalized Exponential Distribution," Mathematics and Statistics, Vol. 10, No. 2, pp. 308 - 313, 2022. DOI: 10.13189/ms.2022.100204.

(b): Nazrina Aziz, Tan Jia Xin, Zakiyah Zain, Mohd Azri Pawan Teh (2022). New Group Chain Sampling Plan (NGChSP-1) for Generalized Exponential Distribution. Mathematics and Statistics, 10(2), 308 - 313. DOI: 10.13189/ms.2022.100204.

Copyright $\bigcirc 2022$ by authors, all rights reserved. Authors agree that this article remains permanently open access under the terms of the Creative Commons Attribution License 4.0 International License

\begin{abstract}
Acceptance criteria are the conditions imposed on any sampling plan to determine whether the lot is accepted or rejected. Group chain sampling plan (GChSP-1) was constructed according to the 5 acceptance criteria; modified group chain sampling plan (MGChSP-1) was derived with 3 acceptance criteria; later new group chain sampling plan (NGChSP-1) was introduced with 4 acceptance criteria where the NGChSP-1 balances the acceptance criteria between the GChSP-1 and MGChSP-1. Producers favor a sampling plan with more acceptance criteria because it reduces the probability of rejecting a good lot (producer risk), whereas consumers may prefer a sampling plan with fewer acceptance criteria as it reduces the probability of accepting a bad lot (consumer risk). The disparity in acceptance criteria creates a conflict between the two main stakeholders in acceptance sampling. In the literature, there are numerous methods available for developing sampling plans. To date, NGChSP-1 was developed using the minimum angle method. In this paper, NGChSP-1 was constructed with the minimizing consumer's risk method for generalized exponential distribution where mean product lifetime is used as quality parameter. There are six phases involved to develop the NGChSP-1 for different design parameters. Result shows the minimum number of groups decrease when the value of design parameters increases. The results of the performance comparison show that the NGChSP-1 is a better sampling plan than the GChSP-1 because it has a smaller number of groups and lower probability of lot acceptance than the GChSP-1. NGChSP-1 should offer better alternatives to industrial practitioners in sectors involving product life test.
\end{abstract}

Keywords Consumer's Risk, Generalized Exponential (GE) Distribution, Minimum Number of Groups, Probability of Lot Acceptance

\section{Introduction}

In the 1930s and 1940s, acceptance sampling was mainly used for receiving inspection in the field of statistical quality control. The purpose of acceptance sampling is to sentence the lots. Acceptance sampling plans do not provide any form of direct quality control. It is a decision-making tool that determines whether an entire lot should be accepted or rejected based on the inspection of a sample. The basic procedure of acceptance sampling is begun by receiving a lot of items from the producer or supplier. The items could be raw materials used in manufacturing or completed products which are ready to be used by the consumer. Next, a small random sample from the submitted lot is drawn, and the sample is inspected based on the quality characteristics of each item in the sample.

There are two classifications of data used to represent the quality characteristics known as sampling plan by attribute and sampling plan by variable [1]. Sampling plan by attribute data considers the quality characteristics that are measured as either defective or non-defective which involves only binary, discrete or integer values. On the other hand, sampling plan by variable deals with the quality characteristics that are measured on a continuous scale such as length and weight of 
a product. Examples of the attribute sampling plans are single sampling plans (SSP), double sampling plans (DSP), multiple sampling plans, sequential sampling plans, group sampling plans (GSP), chain sampling plans (ChSP-1) and group chain sampling plans (GChSP-1).

Acceptance sampling merely inspects a small sample from the entire lot, hence, there is a possibility of making a correct or incorrect decision, which is referred to as a risk. In sampling plan there are 2 risks, which are consumer's risk, $\beta$ and producer's risk, $\alpha$. The $\beta$ is the chances of accepting a bad lot whereas the $\alpha$ is the possibility of rejecting a good lot. In this study, the highlight is on the $\beta$ only. When researchers are trying to establish a novel sampling plan, a low-risk acceptance sampling plan is always required. The operating characteristic (OC) curve is used to ensure that the risk is kept as low as possible. The OC curve is an important tool in acceptance sampling since it has the ability to differentiate between good and bad lots.

The development of various types of sampling plans by many researchers keeps growing from time to time in order to provide more options to industries in assuring their quality of products. The main concern in a sampling plan is to find minimum sample size or minimum number of groups. Epstein developed the SSP for truncated life test for exponential lifetime distribution [2]. Yan and Liu stated that the SSP is the most commonly used in industrial engineering because it is easy to be implemented [3]. Rao realized that the operating characteristics (OC) curve for the SSP changes as the acceptance number, $c$ changes [4]. Sampling plans with lower $c$ values provide protection at lower levels of lot fraction defective than sampling plans with higher $c$ values. Therefore, Dodge suggested chain sampling plan (ChSP-1) to replace SSP with zero acceptance numbers [5]. However, the problem in the ChSP-1 is that it still needs to inspect the items one by one.

If items are tested simultaneously, then time and cost can be reduced. This kind of testing is known as the GSP where several items are placed in one group to be tested. The GSP managed to reduce the time wasting in the SSP, which takes longer inspection time as it inspects the items one by one. Mughal realized that the combination of GSP and ChSP-1 can unravel the problems in the SSP and GSP [6]. Therefore, GChSP-1 was proposed where the lot is accepted if the number of defectives, $d=0$ and the lot is rejected if $d>1$. Besides that, the lot is also accepted if $d=1$ provided that there is no defective in the preceding lot, $d_{i}=0$. Otherwise, the lot is rejected [7-9].

Varied sampling plans have different acceptance criteria. Acceptance criteria are the requirements that must be met by a sampling plan in order to determine whether a lot is accepted or, in the worst-case situation, rejected. Producers favor a sampling plan with more acceptance criteria, whereas consumers may prefer a sampling plan with fewer acceptance criteria. The group chain sampling plan
(GChSP-1) was developed using five acceptance criteria; a modified group chain sampling plan (MGChSP-1) was produced using three acceptance criteria; and recently, a new group chain sampling plan (NGChSP-1) was constructed using four acceptance criteria where the NGChSP-1 balances the acceptance criteria between the GChSP-1 and MGChSP-1.

The new group chain sampling plans (NGChSP-1) are also introduced to tighten the acceptance criteria for the GChSP-1, where the GChSP-1 straight away accepts the current lot with zero defective without considering the number of defectives in the preceding lots, $d_{i}$. The NGChSP- 1 considers the $d_{i}$ in the preceding lots, where the preceding lots must have $d_{i} \leq 1$. If not, the current lot in the NGChSP-1 is rejected. To date, NGChSP-1 was developed using the minimum angle method (MAM) where the performance is only measured by the optimal number of groups [10]. Optimal number of group is selected according to the minimum angle obtained. In this paper, NGChSP-1 was constructed with the minimizing consumer's risk method for Generalized exponential distribution where mean product lifetime is used as quality parameter. The performance of a sampling plan developed by minimizing the consumer's risk is measured based on the minimum number of groups, $g$ (where a lower $g$ reduces inspection resources) and the probability of lot acceptance, $L(p)$ (where a lower $L(p)$ provides better protection to the consumer). NGChSP-1 constructed based on the minimizing consumer's risk method should offer better alternatives to industrial practitioners in sectors involving product life test.

\section{Materials and Methods}

There are six phases to develop the NGChSP-1 as shown below:

\subsection{Phase I: Identifying the Design Parameter}

Design parameter is the pre-specified value in order to construct the NGChSP-1. Besides that, the design parameter is needed to test the hypothesis where the true mean life, $\mu$ is greater than the specified mean life, $\mu_{0}$. The design parameters involved in this study are consumer risk, $\boldsymbol{\beta}$, specified constant, $a$, number of preceding lots, $i$ and number of items, $r$. The design parameters values are given in Table 1.

Table 1. Design Parameter

\begin{tabular}{ccccccccc}
\hline $\boldsymbol{\beta}$ & 0.01 & 0.05 & 0.10 & 0.25 & & & & \\
$a$ & 0.25 & 0.50 & 0.75 & 1.00 & 1.25 & 1.50 & 1.75 & 2.00 \\
$i$ & 1 & 2 & 3 & 4 & & & & \\
$r$ & 2 & 3 & 4 & 5 & & & & \\
\hline
\end{tabular}




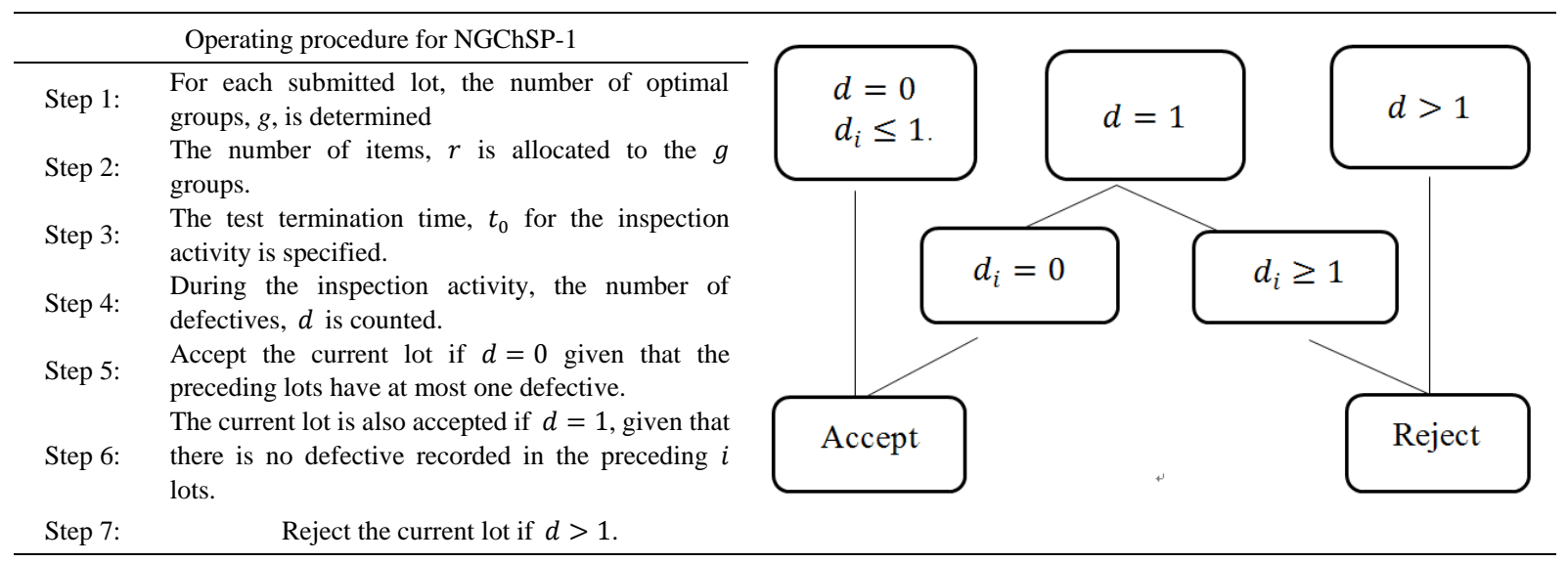

Figure 1. Operating Procedure

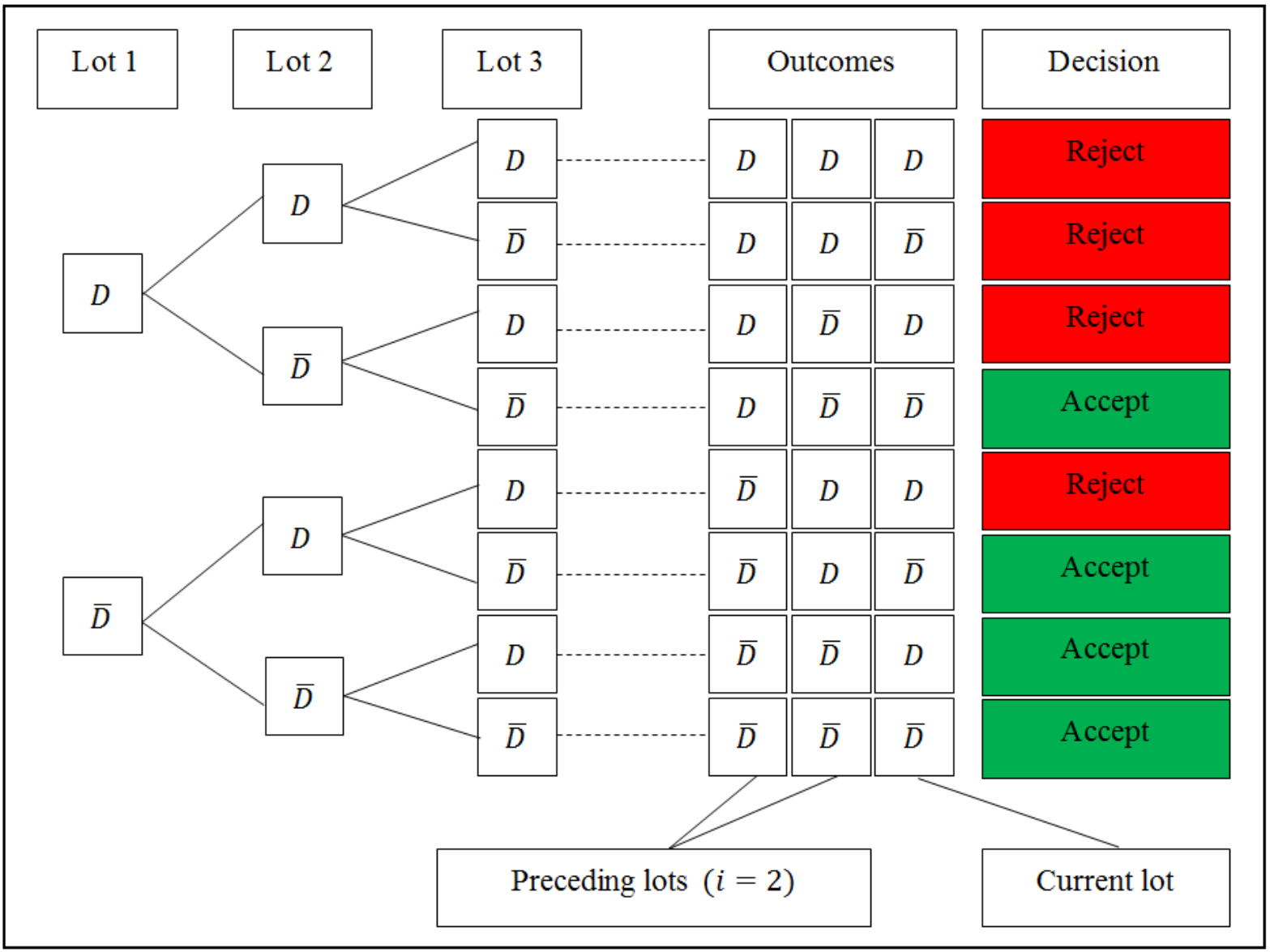

Figure 2. Tree diagram for NGChSP-1

\subsection{Phase II: Constructing Operating Procedure}

The operating procedure for the NGChSP-1 is shown in Figure 1.

\subsection{Phase III: Developing the $L(p)$}

Figure 2 shows all the possible outcomes of NGChSP-1 and a decision either to accept or reject the lot. The decision is based on the operating procedure in Phase II. Next, by using the acceptance decision, the probability of lot acceptance, $L(p)$ is derived where $P_{0}$ represents the probability of having zero defective in the lot and $P_{1}$ represents the probability of having one defective in the lot. Equation (1) shows the $L(p)$ for the NGChSP-1.

$$
\begin{gathered}
L(p)=P_{0} P_{0} P_{0}+P_{0} P_{0} P_{1}+P_{0} P_{1} P_{0}+P_{1} P_{0} P_{0} \\
=P_{0}^{2}\left(P_{0}+3 P_{1}\right) \\
=P_{0}{ }^{i}\left(P_{0}+(i+1) P_{1}\right)
\end{gathered}
$$




\subsection{Phase IV: Deriving $\boldsymbol{P}_{\mathbf{0}}$ and $\boldsymbol{P}_{\mathbf{1}}$}

$P_{0}$ and $P_{1}$ are derived from the binomial distribution as the procedure involves two outcomes which are defective and non-defective. $P_{0}$ and $P_{1}$ in term of the formula of the binomial distribution are shown below, where $n$ denotes the sample size, and $p$ denotes the fraction defective.

$$
\begin{gathered}
P_{0}=(1-p)^{n} \\
P_{1}=n p(1-p)^{n-1}
\end{gathered}
$$

Replacing $n=r g$ in equations (2) and (3),

$$
\begin{gathered}
P_{0}=(1-p)^{r g} \\
P_{1}=r g p(1-p)^{r g-1}
\end{gathered}
$$

In the NGChSP-1, the $g$, is specified by solving the following equation:

$$
L(p) \leq \beta
$$

The $P_{0}$ and $P_{1}$ from equations (4) and (5) are substituted into equation (1) in Phase III, giving the $L(p)$ for the NGChSP-1 as:

$$
L(p)=(1-p)^{r g(i+1)}[(1+(i+1) r g p) /(1-p)]
$$

The CDF of the GE is set by

$$
F(t ; \sigma)=\left[1-\exp \left(-\frac{t}{\sigma}\right)\right]^{\lambda}, t>0, \lambda>0 .
$$

Equation (8) shows the fraction defective, $p$ where it represents the probability that an item will fail at a specified time. Let $t=a \mu_{0}, \sigma=\mu$ and then substitute into equation (8).

Therefore, the fraction defective is given by

$$
p=\left[1-\exp \left(-a\left(1 /\left(\mu / \mu_{0}\right)\right)\right)\right]^{\lambda}
$$

where $\mu$ is the true mean life time, and $\mu_{0}$ is the specified mean life time.

\subsection{Phase V: Deriving the Fraction Defective, $p$}

In this phase, the Generalized Exponential (GE) Cumulative Distribution Function (CDF) is used to derive the fraction defective. CDF is used in this study because we consider time truncation.

\subsection{Phase VI: Measuring Performance}

The NGChSP-1 performance is measured according to the $g$ and the $L(p)$. Apart from that, a comparison of performance

\section{Findings}

There are four different levels of $\beta$ and seven different levels of specified constant, $a$ is used to calculate the $g$, as given in Table 2.

\subsection{Minimum Number of Groups, $g$}

Based on Table 2, the $g$ decreases as the $\beta, i, r$ and

\begin{tabular}{|c|c|c|c|c|c|c|c|c|c|c|}
\hline \multirow{2}{*}{$\beta$} & \multirow{2}{*}{$\mathbf{i}$} & \multirow{2}{*}{$\mathbf{r}$} & \multicolumn{8}{|c|}{$\mathbf{a}$} \\
\hline & & & 0.25 & 0.50 & 0.75 & 1.00 & 1.25 & 1.50 & 1.75 & 2.00 \\
\hline \multirow{4}{*}{0.01} & 1 & 2 & 7 & 4 & 3 & 2 & 2 & 2 & 2 & 1 \\
\hline & 2 & 3 & 4 & 2 & 2 & 1 & 1 & 1 & 1 & 1 \\
\hline & 3 & 4 & 2 & 1 & 1 & 1 & 1 & 1 & 1 & 1 \\
\hline & 4 & 5 & 2 & 1 & 1 & 1 & 1 & 1 & 1 & 1 \\
\hline \multirow{4}{*}{0.05} & 1 & 2 & 5 & 3 & 2 & 2 & 2 & 1 & 1 & 1 \\
\hline & 2 & 3 & 2 & 2 & 1 & 1 & 1 & 1 & 1 & 1 \\
\hline & 3 & 4 & 2 & 1 & 1 & 1 & 1 & 1 & 1 & 1 \\
\hline & 4 & 5 & 1 & 1 & 1 & 1 & 1 & 1 & 1 & 1 \\
\hline \multirow{4}{*}{0.10} & 1 & 2 & 5 & 3 & 2 & 2 & 1 & 1 & 1 & 1 \\
\hline & 2 & 3 & 2 & 1 & 1 & 1 & 1 & 1 & 1 & 1 \\
\hline & 3 & 4 & 2 & 1 & 1 & 1 & 1 & 1 & 1 & 1 \\
\hline & 4 & 5 & 1 & 1 & 1 & 1 & 1 & 1 & 1 & 1 \\
\hline \multirow{4}{*}{0.25} & 1 & 2 & 3 & 2 & 2 & 1 & 1 & 1 & 1 & 1 \\
\hline & 2 & 3 & 2 & 1 & 1 & 1 & 1 & 1 & 1 & 1 \\
\hline & 3 & 4 & 1 & 1 & 1 & 1 & 1 & 1 & 1 & 1 \\
\hline & 4 & 5 & 1 & 1 & 1 & 1 & 1 & 1 & 1 & 1 \\
\hline
\end{tabular}
$a$ increases. For example, when $(i, r, a)=(1,2,0.25)$ and $\beta$ increases from 0.01 to 0.25 , the $g$ is decreased from 7 to 3 . The $g$ also decreases from 7 to 2 when $(\beta, a)=$ $0.01,0.25), i$ increases from 1 to 4 and $r$ increases from 2 to 5 . Besides, the $g$ decreases from 7 to 1 when $(\beta, i, r)=$ $(0.25,4,5)$ and $a$ increases from 0.25 to 2.00 .

Table 2. The $g$ for NGChSP-1 based on GE distribution with $\lambda=1$ 
Table 3. The $\boldsymbol{L}(\boldsymbol{p})$ for NGChSP-1 based on GE distribution with $\lambda=1$

\begin{tabular}{|c|c|c|c|c|c|c|c|c|c|}
\hline \multirow{2}{*}{$\boldsymbol{\beta}$} & \multirow{2}{*}{$g$} & \multirow{2}{*}{$a$} & \multicolumn{7}{|c|}{ Mean Ratio, $\mu / \mu_{0}$} \\
\hline & & & 1 & 2 & 4 & 6 & 8 & 10 & 12 \\
\hline \multirow{8}{*}{0.01} & 7 & 0.25 & 0.00816 & 0.14278 & 0.48758 & 0.68238 & 0.78738 & 0.84858 & 0.88697 \\
\hline & 4 & 0.50 & 0.00382 & 0.10155 & 0.42365 & 0.63012 & 0.74750 & 0.81793 & 0.86293 \\
\hline & 3 & 0.75 & 0.00178 & 0.07176 & 0.36624 & 0.57964 & 0.70755 & 0.78655 & 0.83795 \\
\hline & 2 & 1.00 & 0.00495 & 0.11337 & 0.44284 & 0.64605 & 0.75974 & 0.82738 & 0.87036 \\
\hline & 2 & 1.25 & 0.00095 & 0.05354 & 0.32298 & 0.53886 & 0.67413 & 0.75974 & 0.81630 \\
\hline & 2 & 1.50 & 0.00018 & 0.02463 & 0.23101 & 0.44284 & 0.59126 & 0.69114 & 0.75974 \\
\hline & 2 & 1.75 & 0.00003 & 0.01112 & 0.16278 & 0.35969 & 0.51370 & 0.62388 & 0.70253 \\
\hline & 1 & 2.00 & 0.00891 & 0.14420 & 0.48651 & 0.68073 & 0.78583 & 0.84726 & 0.88587 \\
\hline \multirow{8}{*}{0.05} & 5 & 0.25 & 0.04501 & 0.30067 & 0.65606 & 0.80441 & 0.87508 & 0.91362 & 0.93681 \\
\hline & 3 & 0.50 & 0.02177 & 0.21948 & 0.57964 & 0.75152 & 0.83795 & 0.88647 & 0.91620 \\
\hline & 2 & 0.75 & 0.02463 & 0.23101 & 0.59126 & 0.75974 & 0.84378 & 0.89076 & 0.91947 \\
\hline & 2 & 1.00 & 0.00495 & 0.11337 & 0.44284 & 0.64605 & 0.75974 & 0.82738 & 0.87036 \\
\hline & 2 & 1.25 & 0.00095 & 0.05354 & 0.32298 & 0.53886 & 0.67413 & 0.75974 & 0.81630 \\
\hline & 1 & 1.50 & 0.03700 & 0.27224 & 0.62922 & 0.78583 & 0.86203 & 0.90408 & 0.92957 \\
\hline & 1 & 1.75 & 0.01825 & 0.19917 & 0.55526 & 0.73324 & 0.82459 & 0.87647 & 0.90849 \\
\hline & 1 & 2.00 & 0.00891 & 0.14420 & 0.48651 & 0.68073 & 0.78583 & 0.84726 & 0.88587 \\
\hline \multirow{8}{*}{0.10} & 5 & 0.25 & 0.04501 & 0.30067 & 0.65606 & 0.80441 & 0.87508 & 0.91362 & 0.93681 \\
\hline & 3 & 0.50 & 0.02177 & 0.21948 & 0.57964 & 0.75152 & 0.83795 & 0.88647 & 0.91620 \\
\hline & 2 & 0.75 & 0.02463 & 0.23101 & 0.59126 & 0.75974 & 0.84378 & 0.89076 & 0.91947 \\
\hline & 2 & 1.00 & 0.00495 & 0.11337 & 0.44284 & 0.64605 & 0.75974 & 0.82738 & 0.87036 \\
\hline & 1 & 1.25 & 0.07386 & 0.36716 & 0.70691 & 0.83725 & 0.89735 & 0.92957 & 0.94874 \\
\hline & 1 & 1.50 & 0.03700 & 0.27224 & 0.62922 & 0.78583 & 0.86203 & 0.90408 & 0.92957 \\
\hline & 1 & 1.75 & 0.01825 & 0.19917 & 0.55526 & 0.73324 & 0.82459 & 0.87647 & 0.90849 \\
\hline & 1 & 2.00 & 0.00891 & 0.14420 & 0.48651 & 0.68073 & 0.78583 & 0.84726 & 0.88587 \\
\hline \multirow{8}{*}{0.25} & 3 & 0.25 & 0.21948 & 0.57964 & 0.83795 & 0.91620 & 0.94909 & 0.96587 & 0.97554 \\
\hline & 2 & 0.50 & 0.11337 & 0.44284 & 0.75974 & 0.87036 & 0.91947 & 0.94526 & 0.96042 \\
\hline & 2 & 0.75 & 0.02463 & 0.23101 & 0.59126 & 0.75974 & 0.84378 & 0.89076 & 0.91947 \\
\hline & 1 & 1.00 & 0.14420 & 0.48651 & 0.78583 & 0.88587 & 0.92957 & 0.95231 & 0.96561 \\
\hline & 1 & 1.25 & 0.07386 & 0.36716 & 0.70691 & 0.83725 & 0.89735 & 0.92957 & 0.94874 \\
\hline & 1 & 1.50 & 0.03700 & 0.27224 & 0.62922 & 0.78583 & 0.86203 & 0.90408 & 0.92957 \\
\hline & 1 & 1.75 & 0.01825 & 0.19917 & 0.55526 & 0.73324 & 0.82459 & 0.87647 & 0.90849 \\
\hline & 1 & 2.00 & 0.00891 & 0.14420 & 0.48651 & 0.68073 & 0.78583 & 0.84726 & 0.88587 \\
\hline
\end{tabular}

\subsection{The Probability of Lot Acceptance, $L(p)$}

The $L(p)$ for seven levels of mean ratio and four different values of $\beta$ based on $i=1, r=2$ as control design parameters are shown in Table 3. Based on Table 3, as the $\mu / \mu_{0}$ increases, so does the $L(p)$. For example, the $L(p)$ increases from 0.00816 to 0.88697 when $(\beta, g, a)=$ $(0.01,7,0.25)$ and the $\mu / \mu_{0}$ increases from 1 to 12 . It means that the $L(p)$ increases from $0.82 \% 88.7 \%$ when the true average lifetime, $\mu$ of an item is 12 times higher than the stated average lifetime, $\mu_{0}$.

\subsection{Comparison between NGChSP-1 and GChSP-1}

The comparison between NGChSP-1 and GChSP-1 is measured based on the $g$ and the $L(p)$.

\subsubsection{The $\boldsymbol{g}$ for NGChSP-1 and GChSP-1}

Table 4 shows the $g$ for NGChSP-1 and GChSP-1 with $\lambda=1$. The NGChSP-1 has a lower $g$ than the GChSP-1. For example, the NGChSP-1 needs 7 groups while GChSP-1 needs 10 groups, for design parameters $(\lambda, \beta, i, r, a)=(1,0.01,1,2,0.25)$. A smaller $g$ reduces the time and cost, and thus the NGChSP-1 is better compared to the GChSP-1. 
Table 4. The $\mathrm{g}$ for NGChSP-1 and GChSP-1 with $\lambda=1$

\begin{tabular}{|c|c|c|c|c|c|}
\hline \multirow{2}{*}{$\beta$} & \multirow{2}{*}{$\mathbf{i}$} & \multirow{2}{*}{$\mathbf{r}$} & \multirow{2}{*}{$\mathbf{a}$} & \multicolumn{2}{|c|}{ g } \\
\hline & & & & NGChSP-1 & GChSP-1 \\
\hline \multirow{8}{*}{0.01} & \multirow{8}{*}{1} & \multirow{8}{*}{2} & 0.25 & 7 & 10 \\
\hline & & & 0.50 & 4 & 5 \\
\hline & & & 0.75 & 3 & 4 \\
\hline & & & 1.00 & 2 & 3 \\
\hline & & & 1.25 & 2 & 2 \\
\hline & & & 1.50 & 2 & 2 \\
\hline & & & 1.75 & 2 & 2 \\
\hline & & & 2.00 & 1 & 2 \\
\hline
\end{tabular}

3.3.2. The $\boldsymbol{L}(\boldsymbol{p})$ for NGChSP-1 and GChSP-1

Table 5 shows the $L(p)$ for NGChSP-1 and GChSP-1 with $\lambda=1$.

Table 5. The $\boldsymbol{L}(\boldsymbol{p})$ for NGChSP-1 and GChSP-1 when $\lambda=1$

\begin{tabular}{ccccccc}
\hline \multirow{\beta}{*}{} & $\mathbf{i}$ & $\mathbf{r}$ & $\mathbf{a}$ & $\boldsymbol{\mu} / \boldsymbol{\mu}_{\mathbf{0}}$ & \multicolumn{2}{c}{$\mathbf{L}(\mathbf{p})$} \\
& & & & NGChSP-1 & GChSP-1 \\
\hline & & & & 0.00816 & 0.03382 \\
& & & & 2 & 0.14278 & 0.23006 \\
& & & & 4 & 0.48758 & 0.57377 \\
0.01 & \multirow{2}{*}{1} & 2 & \multirow{2}{*}{0.25} & 6 & 0.68238 & 0.74352 \\
& & & & 8 & 0.78738 & 0.83091 \\
& & & 10 & 0.84858 & 0.88068 \\
& & & 12 & 0.88697 & 0.91149 \\
\hline
\end{tabular}

Based on Table 5, when compared to GChSP-1, NGChSP-1 has a smaller $L(p)$. For example, the $L(p)$ for NGChSP-1 is 0.00816 while the $L(p)$ for GChSP-1 is 0.03382 for design parameters $\left(\lambda, \beta, i, r, a, \mu / \mu_{0}\right)=$ $(1,0.01,1,2,0.25,1)$.

\section{Conclusion}

This study focuses on the attribute acceptance sampling plan particularly the NGChSP-1. The NGChSP-1 is developed for the GE distribution by minimizing the $\beta$ only. Four design parameters with different values are used to construct the NGChSP-1. The $L(p)$ is derived based on the acceptance criteria for the NGChSP-1. The performances of the NGChSP-1 are measured according to the $g$ and the $L(p)$. Furthermore, the performances comparisons between the NGChSP-1 and the GChSP-1 are made. Findings reveal that the NGChSP-1 is a better sampling plan compared to the GChSP-1, as it has smaller $g$ and lower $L(p)$ compared to the GChSP-1.

\section{Acknowledgements}

This research was supported through Fundamental Research Grant Scheme (FRGS/1/2018/STG06/UUM/02/6) by Ministry of Higher Education (MoHE) of Malaysia.

\section{REFERENCES}

[1] Montgomery, D.C. Introduction to Statistical Quality Control. John Wiley \& Sons. 2019.

[2] Epstein, B. (1954). Truncated life tests in the exponential case. The Annals of Mathematical Statistics, Volume 25, Number 3, PP: 555-564. 1954.

[3] Yan \& Liu. Designing a repetitive group sampling plan for Weibull distributed processes. Mathematical Problems in Engineering, Volume 2016, PP:1-10. 2016.

[4] Dodge, H. F. Chain sampling inspection plan. Industry Quality Control, Volume 11, Number 4, PP: 10-13. 1955.

[5] Rao, G. S. A group acceptance sampling plans based on truncated life tests for Marshall - Olkin extended Lomax distribution. Electronic Journal of Applied Statistical Analysis, Volume 3, Number 1, PP: 18-27. 2010.

[6] Mughal, A. R. A family of group chain acceptance sampling plans based on truncated life test. (Unpublished Doctoral dissertation.) Universiti Utara Malaysia, Sintok, Kedah, Malaysia. 2018.

[7] Teh, M. A. P., Aziz, N., \& Zain, Z. Time truncated group chain sampling plans for gamma distribution. International Journal of Innovative Technology and Exploring Engineering, Volume 8, Number 5S, PP: 62-65. 2019

[8] Teh, M. A. P., Aziz, N., \& Zain, Z. Group chain sampling plans based on truncated life test for inverse Rayleigh distribution. Research Journal of Applied Sciences, Volume 11, Number 11, PP: 1432-1435. 2016.

[9] Aziz, N., Hasim, Z. and Zain, Z. Two -Sided Group Chain Sampling Plans Based on Truncated Life Test for Generalized Exponential Distribution. Mathematics and Statistics, Volume 9, Number 4, PP: 439 - 444. 2021. DOI: 10.13189/ms.2021.090404

[10] Teh, M. A. P., Aziz, N., \& Zain, Z. A new method in designing group chain acceptance sampling plans (GChSP) for generalized exponential distribution. International Journal of Quality and Reliability Management, Volume 38, Number 5, PP: 1116-1129. 2021. 